\title{
3-D Scanning Transmission Electron Microscopy of Carbide-Derived Carbons for Electrical Energy Storage
}

\author{
Scarlett Widgeon ${ }^{1}$, Mhairi Gass ${ }^{2}$, Andrew Bleloch ${ }^{2}$, Sun-Hwa Yeon ${ }^{3}$, Yury Gogotsi ${ }^{3}$, and Ilke \\ Arslan $^{1}$
}
1. University of California-Davis, Chemical Engineering and Materials Science Dept., One Shields Ave., Davis, CA 95616, USA.
2. UK SuperSTEM Laboratory, Daresbury Laboratory, Daresbury, WA4 4AD, UK.

3. Drexel University, Materials Science and Engineering Dept., 3141 Chestnut St., Philadelphia, PA 19104, USA.

Within the next 50 years, the world energy consumption is predicted to double. The United States is dependant on fossil fuels as its primary source of energy, which has become increasingly problematic due to global warming and national security issues. This increasing demand for energy has raised awareness for clean, efficient, and renewable energy sources, such as wind and solar energy. However, the use of these sources requires efficient electrical energy storage (EES). This is necessary to provide constant and reliable electricity, and for the advancement of hybrid electric vehicles to all-electric or plug-in hybrid vehicles [1], but the materials commercially available today cannot store the energy necessary for these desired applications. Synthesized nanostructured electrodes, such as carbide-derived carbons (CDCs) can be engineered to have high surface areas that may store multiple charges at a single site, thus increasing the charge density. CDCs have shown promising specific capacitance when used with several different electrolytes systems in supercapacitors and they offer more control of their physical properties than other conventional carbon structures [2].

CDCs have been studied here to gain a fundamental understanding of the non-periodic structures and pore surfaces using a combination of atomic-resolution imaging, atomic-resolution electron energy-loss spectroscopy (EELS), and three dimensional imaging in the scanning transmission electron microscope (STEM). Figure 1 shows a schematic illustrating how an aberrationcorrected STEM probe can be used to gain a 3-D understanding of a nanomaterial with atomic resolution. During synthesis of the $\mathrm{CDCs}$, impurity atoms from the precursors remain in the mesoporous carbon structure that cannot be removed. Our preliminary results (Figure 2) show that in some materials, the impurity atoms segregate to the surfaces of the pores, with large pore volumes of $1.5-2.2 \mathrm{~cm}^{3} / \mathrm{g}$ and average pore sizes in the range of 5-6 nm or 15-16 nm, depending on the material. This segregation will obviously alter the surface chemistry and ability of the surfaces to retain charge. Results will be discussed from mesoporous CDC samples produced by chlorination of $\mathrm{SiCN}$ and $\mathrm{Ti}_{2} \mathrm{AlC}$ precursors [3], [4].

References

[1] J.B. Goodenough, H.D. Abruña, M.V. Buchanan, Basic Research Needs for Electrical Energy Storage: Report of the Basic Energy Sciences Workshop for Electrical Energy Storage (2007).

[2] P. Simon, Y. Gogotsi, Nature Materials 7 (2008) 845.

[3] J. Chmiola, G. Yushin, Y. Gogotsi, C. Portet, P. Simon, P. L. Taberna, Science 313 (2006) 1760 . 
[4] This work was supported in part by Sandia's Truman Fellowship LDRD. Sandia is a multiprogram laboratory operated by Sandia Corporation, a Lockheed Martin Company, for the United States Department of Energy under contract DE-AC04-94AL85000.
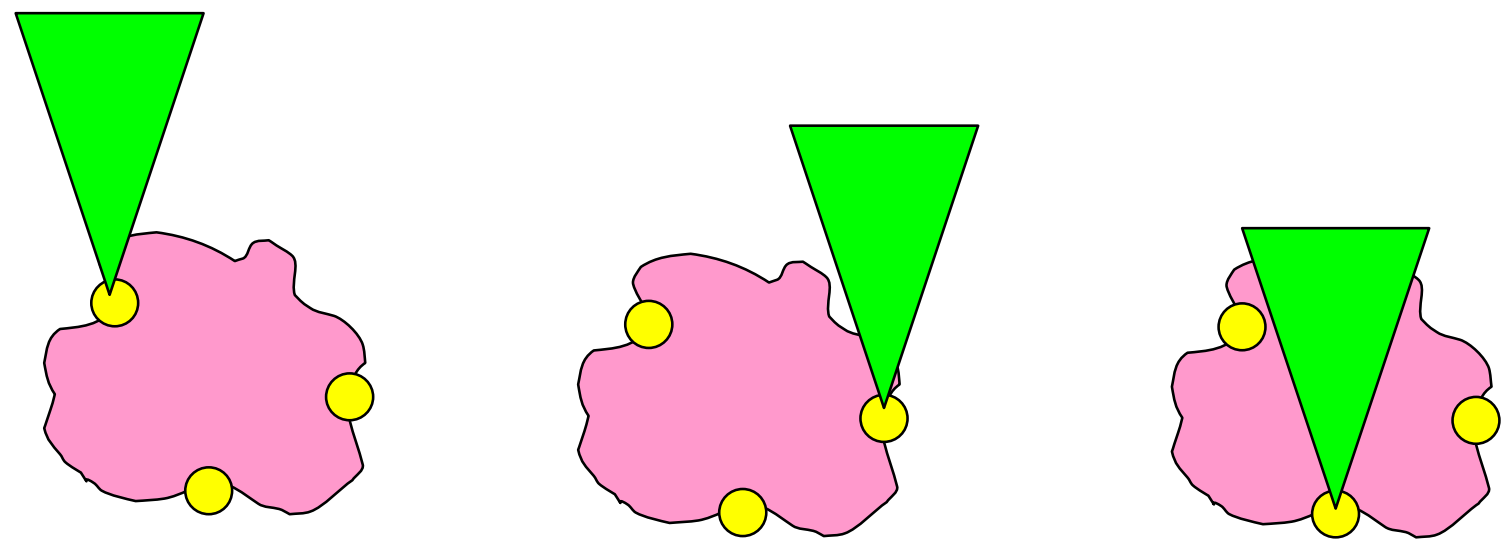

Figure 1: Schematic of STEM probe scanning through a three-dimensional object. The aberration-corrected probe focuses at different $z$-heights through the object, thus allowing single atoms to be imaged through the thickness of the material for an atomic-scale 3-D representation.
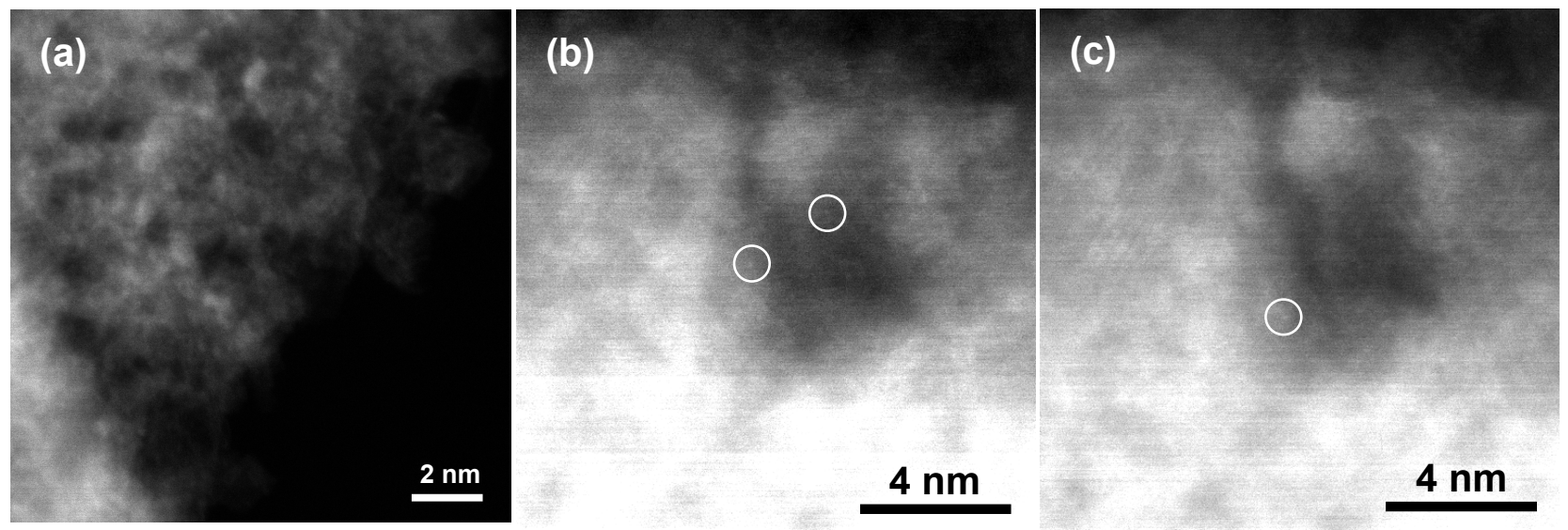

Figure 2: (a) is a low magnification image showing the porosity of the CDC material. (b) and (c) are two images taken through the thickness of the material, showing single atoms at different heights surrounding one pore. Some of the atoms are indicated by circles. The contrast and brightness in (b) and (c) were enhanced to highlight the atoms. 